\title{
Extraction Rate in Vapor Assisted Extraction of Heavy Oil (VAPEX)
}

\author{
Vijitha Mohan and Parthasakha Neogi ${ }^{\star}$, Missouri University of Science and Technology, Rolla, \\ USA
}

\section{Abstract}

Heavy oil recovery requires either heat (SAGD) or a solvent (VAPEX) to reduce its high viscosity first and then the less viscous oil can be recovered. We show here that the existing data, on the rate of oil recovered in VAPEX process in sandpacks show a square root dependence on the height of pay zone, following expectation. This dependence had remained uncertain and is of importance in oil field operations. We have used dimensional analysis and inverse viscosity-diffusivity dependence to obtain an expression for the rate that agrees well with the available sandpack data. The dimensional analysis overcomes the uncertainty in the number of independent variables and leads to standard dimensionless groups. The inverse dependence is based on the free volume theory which we have tested earlier successfully for heavy oils. The final correlation is shown to work well in the limit where we go from sandpacks to reservoir.

\section{Introduction}

Highly viscous heavy oil (above $100 \mathrm{mPa}$ s) cannot be recovered from underground reservoirs without the aid of an external resource like heat or solvents, which reduces its viscosity before recovery. Steam assisted gravity drainage (SAGD) process is a thermal process where steam is used as a heat source to heat up the heavy oil, reducing the viscosity to $\sim 5-10 \mathrm{mPa}$. The less viscous oil flows under gravity to the drainage well. The alternate, which requires no water, is the vapor assisted petroleum extraction (VAPEX) process that uses gases (above critical temperature) or vapors (below critical temperature) which form a part of products on condensation. They dissolve in the oil at the interface and diffuse into the bulk. In the process, the viscosity of the solution is brought down and it drains under gravity (Banerjee 2012).

The rate of oil recovery that has been predicted by theory, is not fully backed by the experimental data. Specifically, it is the role of $h$, the height of the pay zone, which does not appear to conform to any pattern. The results by Mokrys and Butler (1993) provides the recovery rate,

$$
Q_{b}=\sqrt{2 k g \phi \Delta S_{o} h N_{s}}
$$

in $\mathrm{m}^{3} /(\mathrm{m}$-width $\cdot \mathrm{s})$, where $\Delta S_{o}$ is the difference between the fractional pore volume containing oil before and after displacement. For perfect displacement, $\Delta S_{o}=1$. Others parameters in the Eq. 1 are permeability $k$, acceleration due to gravity $g$, and porosity $\phi . N_{s}$ is defined as,

$$
N_{s}=\int_{\varphi_{\min }}^{1} \frac{\Delta \rho D(1-\varphi)}{\mu} d \ln \varphi
$$

where $\Delta \rho$ is the density difference between pure oil and displacing fluid (vapor). $\varphi$ is the volume fraction of the solvent in oil, and $\varphi_{\min }$ is the solvent concentration at the end of the front of the solvent that has penetrated the oil. Mohan et al. (2019) improved the earlier model using mass transfer boundary layer theory. But their final results are not that different.

Copyright ( $\odot$ the author(s). This work is licensed under a Creative Commons Attribution 4.0 International License. DOI: $10.14800 /$ IOGR.430 


$$
Q_{b}=2 \sqrt{\frac{k g \phi \Delta \rho h D_{o}}{\mu_{o}}} \int_{0}^{u} e^{\alpha \varphi_{o} \psi}\left(1-\varphi_{o} \psi\right) d \psi
$$

The terms under the square-root sign (only) also appears in Eq. 1 if we express $D=D_{o} \times$ function of $\varphi$, and $\mu$ $=\mu_{o} \times$ function of $\varphi$. Here,

$$
u=\frac{\delta}{\sqrt{4 \mu_{o} D_{o} \eta \phi /(k \Delta \rho g \sin \theta)}}
$$

where $\delta$ is the thickness over which solvent concentration in oil falls from volume fraction of $\varphi_{o}$ to $\varphi_{\min }$ in the direction normal to the interface, $\eta$ is the direction tangential to the interface, and $u$ is taken to be a constant. If we use a vapor which can condense and the condensate is miscible in oil, then $\varphi_{o}=1$. For most solvents, known to us $\bar{\alpha}=\alpha \varphi_{o} \sim 10$ where the free volume theory (Mohan et al. 2017) is used to write the diffusivity as,

$$
D=D_{o} e^{\alpha \varphi}
$$

and the viscosity as,

$$
\mu=\mu_{o} e^{-\alpha \varphi}
$$

Mokrys and Butler (1993) conducted experiments in vertically held Hele-Shaw cells and verified the above $k$ and $h$ dependence. The vapor is introduced from the side as shown in Figure 1.

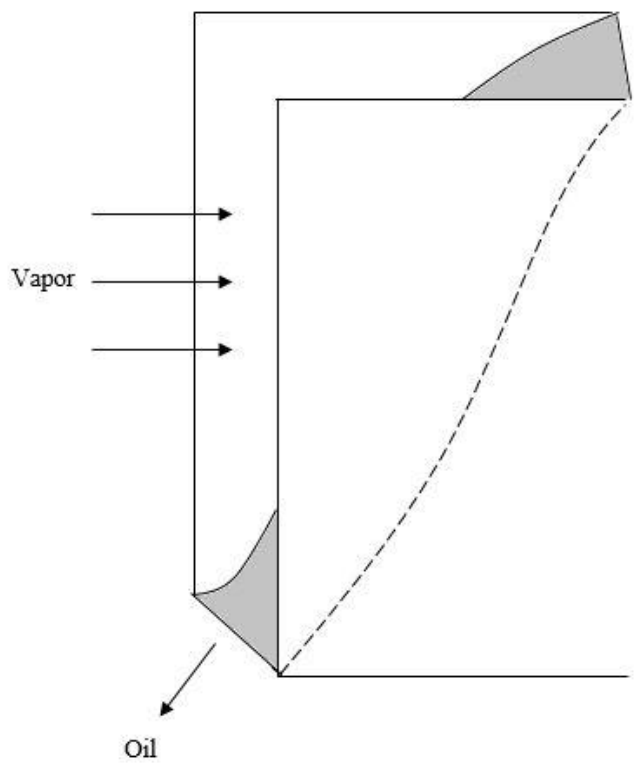

Figure 1-Schematic view of the recovery experiments.

In Hele-Shaw cells the gap between the two parallel plates is empty and then is filled with oil. In sandpack experiments the gap is filled with sand and oil mixture which is then packed. Other experiments using twodimensional sandpacks followed and showed a result that was proportional to $h$ rather than $h^{1 / 2}$ as in above (Karmakar and Maini 2003; Yazdani and Maini 2005; Haghighat and Maini 2012). One numerical simulation also showed such a result (Cuthiell and Edmunds 2013). Nenninger and Dunn (2008) put together a large number of data from sandpacks and Hele-Shaw cell and found,

$$
W_{b}=43550\left(k \phi / \mu_{o}\right)^{0.51}
$$

where,

$$
W_{b}=Q_{b} \rho_{o} / h
$$


Note that the terms under the square-root appear in both Eqs. 1 and 2. However, the correlation failed to show an $h$ dependence although some dependence is seen. Thus, we face a situation where we cannot be sure what the experiments have to say regarding $h$, an important field variable.

\section{Formulation}

We note that Eq. 7 is somewhat along the lines of dimensional analysis. However, if we try to fit variables

$$
y=\mathrm{M} x_{1}^{m 1} x_{2}^{m 2}
$$

there is no reason to suppose that the constants $M, m_{1}, m_{2}$, etc. are independent of one another. This problem is eliminated by Buckingham-pi theorem which gives us the correct number of independent variables. We take $W_{b}$ to be a function of $\mu_{o}, \phi, \Delta \rho, k, h$ from Nenninger and Dunn (2008) where we have omitted surface tension. Because of the way $\phi$ is associated with $k$ in Eq. 7, $\phi$ has been considered only as a product $\phi k$. We now add to these all mass transfer variables $D_{o}, \alpha, \varphi_{o}$. Since $\alpha \varphi_{o} \sim 10$, it is not considered to be a variable here. Similarly, most of the data compiled have $\varphi_{o}=1$ and it is not considered to be a variable. Hence, there are seven variables and three dimensions leading to four dimensionless groups. These groups are found to be

$$
\begin{aligned}
R e & =W_{b} h / \mu_{o} \\
F r & =\frac{\phi k \Delta \rho}{\mu_{o}} \sqrt{\frac{g}{h}}, \\
A r & =\phi k / h^{2}, . \\
S c & =\frac{\mu_{o}}{\Delta \rho D_{o}}, \ldots .
\end{aligned}
$$

where $R e$ is the Reynolds' number, which is the ratio between the inertial and the viscous forces; $F r$ is the Froude number, which is the square-root of the ratio between kinetic energy and potential energy due to gravity; $A r$ is a square of aspect ratio; and $S c$ is Schmidt's number. Note that $h$ plays an important role of providing a length scale. Finally, $\Delta \rho \sim \rho_{o}$ where two values of specific gravities of oil, 0.8 and 0.9 are used. Diffusivity at infinite dilution $D_{\mathrm{o}}$ is difficult to find, and Stokes-Einstein's equation is used to calculate this value following Mohan et al. (2017). Note the inverse relation $D_{o}$ is defined as

$$
D_{o}=B / \mu_{o}
$$

where $B$ is a constant. Since $\operatorname{Re}$ (flow rate) and $\operatorname{Fr}$ (gravity) are the two important variables, we plotted $\operatorname{Re}$ versus $F r$ and found that the data compiled by Nenninger and Dunn (2008) lay on a straight lines. Thus, we take

$$
\operatorname{Re}=\Lambda F r^{a} A r^{b} S c^{c},
$$

where $\Lambda, a, b$ and $c$ are unknown and set $a$ to 1 . We look at how $W_{b}$ is affected by $\phi k$ from Eq. 7 and set the net power on it to $1 / 2$. Similarly, the power on $\mu_{o}$ is set to $-1 / 2$. As a result, $b$ and $c$ were calculated to be $-1 / 2$ and $-1 / 4$. $\Lambda$ can be determined by fitting to the data (Figure 2 ) and the result is shown below.

$$
\frac{F r}{R e}=4 \times 10^{-6} A r^{1 / 2} S c^{1 / 4}
$$

\section{Results and Discussion}

Eq. 16 and the data (Nenninger and Dunn 2008) have been plotted in Figure 2 using the specific gravity of heavy oils to be 0.9 . There are 6 outliers in their group of 43 on sandpacks that have been omitted. Of these, three points are outliers to Eq. 7 as well and the other three have combined heat and mass transfer. The fit has been stretched in Figure 3 to show that it is excellent at small values. This is good, as the rock data will show at even smaller values of $\sqrt{k} / h$. 


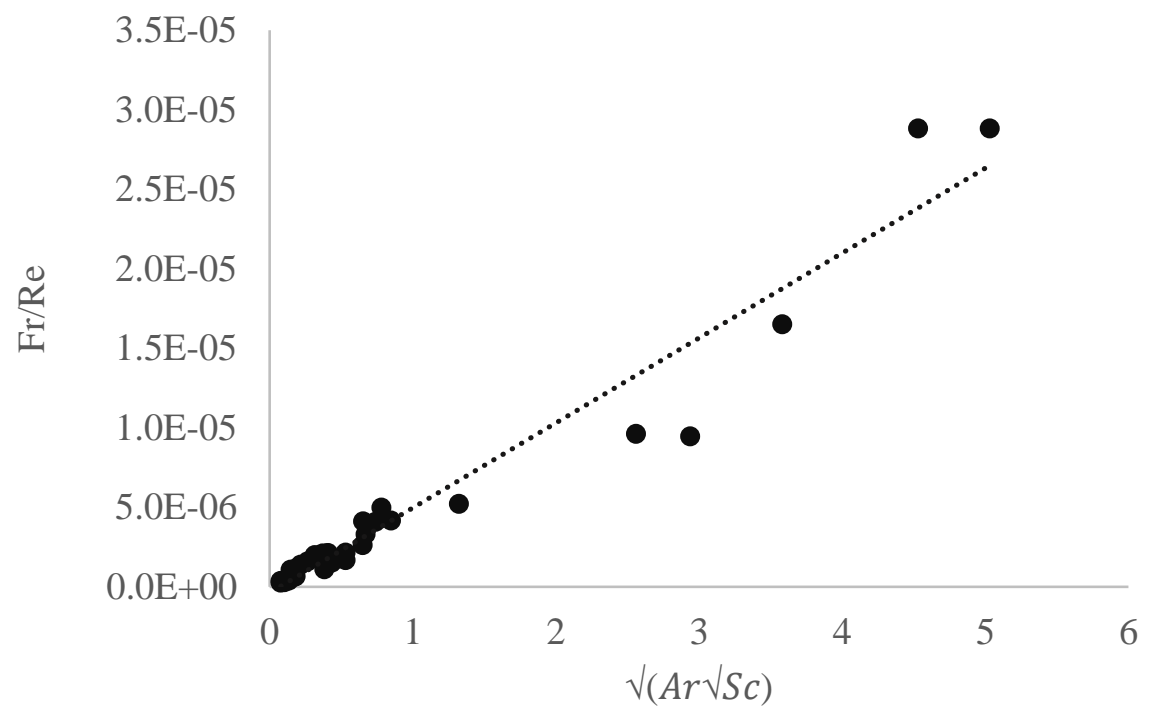

Figure 2-Plots of Fr/Re against $\sqrt{A r \sqrt{S c}}$ in sandpacks from the compilation by Nenninger and Dunn (2008). Specific gravity of heavy oil is taken to be 0.9 .

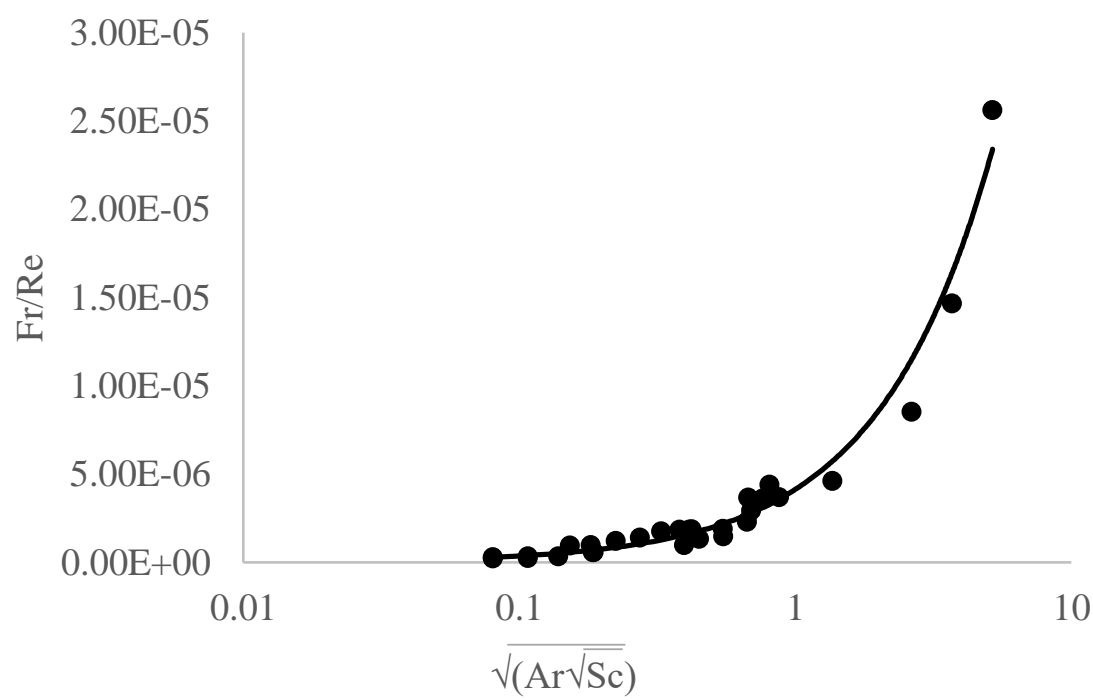

Figure 3-Plots of $F r / R e$ against $\sqrt{A r \sqrt{S c}}$ in logscale. Same as in Figure 2 to show the fit at small values of $A r$.

There is more scatter at small values of $h$ (or large $A r$ ) than large values. Nenninger and Dunn (2008) have also compiled data on Hele-Shaw cells (higher $A r$ values), which however had too much scatter and were not considered. Eq. 16 can be re-expressed as

$$
W_{b}=25 \times 10^{4} \sqrt{\frac{\phi k}{\mu_{o}}} \frac{(\Delta \rho)^{5 / 4} g^{1 / 2} B^{1 / 4}}{h^{1 / 2}},
$$

or in terms of $Q_{b}$,

$$
Q_{b}=25 \times 10^{4} \sqrt{\frac{\phi k(\Delta \rho)^{1 / 2} g h D_{o}^{1 / 2}}{\mu_{o}^{1 / 2}}}
$$

which supports the result that $Q_{b} \propto h^{1 / 2}$. 


\section{Conclusions}

Thus, the sandpack data does indeed agree with the result that for small values of $\sqrt{k} / h$, we should see a $h^{1 / 2}$ dependence. However, if we had done a curve fit using Eq. 18 then we may not have obtained the $h$ dependence fully as Nenninger and Dunn (2008) had experienced. Eq. 2 can be expressed as Eq. 16 if the inverse dependence between diffusivity and viscosity with $B$ and $\Delta \rho \sim \rho_{\mathrm{o}}$ are taken to be constants.

\section{Conflicts of Interest}

The author(s) declare that they have no conflicting interests.

\section{Nomenclature}

$$
\begin{aligned}
& D=\text { Diffusivity } \\
& D_{o}=\text { Diffusivity at infinite dilution } \\
& F_{r}=\text { Froude number } \\
& g=\text { Acceleration due to gravity } \\
& h=\text { Total pay zone height of the system } \\
& k=\text { Permeability } \\
& Q_{b}=\text { Recovery rate } \\
& R_{e}=\text { Reynolds' number } \\
& S_{c}=\text { Schmidt's number } \\
& W_{b}=\text { Mass flux }
\end{aligned}
$$

\section{Greek Letters}

$$
\begin{aligned}
& \alpha=\text { Concentration dependence term } \\
& \Delta \rho=\text { Density difference between oil and the vapor } \\
& \Delta S_{o}=\text { Difference in fractional pore volume } \\
& \mu \quad=\text { Viscosity } \\
& \mu_{o}=\text { Viscosity of pure oil } \\
& \rho=\text { Total density } \\
& \rho_{o}=\text { Density of pure oil } \\
& \varphi=\text { Volume fraction of solvent } \\
& \varphi_{o}=\text { Solubility of solvent in oil } \\
& \phi=\text { Porosity }
\end{aligned}
$$

\section{References}

Banerjee, D. K. 2012. Oil Sands, Heavy Oil and Bitumen, From Recovery to Refinery. Tulsa, OK, US: Penn Well Corp. Mokrys, I. J. and Butler, R. M. 1993. The Rise of Interfering Solvent Chambers: Solvent Analog Model of SteamAssisted Gravity Drainage. Journal of Canadian Petroleum Technology 32(3): 26-36.

Cuthiell, D. and Edmunds, N. 2013. Thoughts on Simulating the VAPEX Process. Journal of Canadian Petroleum Technology 52(3):192-203. SPE-158499-PA.

Haghighat, P. and Maini, B. B. 2012. Experimental Evaluation of Heated VAPEX Process. Paper presented at SPE Heavy Oil Conference, Alberta, Canada, 12-14 June. SPE-157799-MS.

Karmakar, K. and Maini, B. B. 2003. Experimental Investigation of Oil Drainage Rates in VAPEX Process for Heavy Oil and Bitumen Reservoirs. Paper presented at SPE Annual Technical Conference and Exhibition, Denver, Colorado, 5-8 October. SPE-84199-MS.

Mohan, V., Neogi, P., and Bai, B. 2019. Revisiting Butler-Mokrys Model for VAPEX Process. Soc. Pet. Eng, J. 24(2): 511-521. SPE-194212-PA.

Mohan, V., Neogi, P., and Bai, B. 2017. Concentration Dependent Diffusivities of Model Solvents in Heavy Oil. Diffusion Fundamentals 27(3):1-27. 
Nenninger, J. E. and Dunn, S. G. 2008. How Fast Is Solvent Based Gravity Drainage? Paper presented at Canadian International Petroleum Conference, Calgary, Alberta, Canada, 17-19 June. PETSOC-2008-139.

Yazdani, A. and Maini, B. 2005. Effect of Drainage Height and Grain Size on Production Rates in the VAPEX Process: Experimental Study. Soc. Pet. Eng. Res. Eval. Eng. 8(3): 205-212. SPE-89409-PA.

Vijitha Mohan received her $\mathrm{PhD}$ in Chemical Engineering from Missouri University of Science and Technology and served as a Lecturer after graduation. Her research interests lie in heavy oil recovery. She acquired her B.Tech in Chemical Engineering from University of Madras and M.S in Chemical Engineering from Mississippi State University. She is now employed in Alberta, Canada.

Parthasakha Neogi, SPE, is a Professor of Chemical Engineering at Missouri University of Science and Technology, where he has worked as faculty for the last 36 years. His research interests are in wetting, surfactants and polymers, and in interfacial transport phenomena. He holds B.Tech. (Hons.) from the Indian Institute of Technology Kharagpur, M. Tech. from the Indian Institute of Technology Kanpur, and Ph.D. from Carnegie-Mellon University, all in chemical engineering. 